\section{P2-166 RELATIONSHIPS OF RISK FACTORS FOR PRE-ECLAMPSIA WITH BLOOD PRESSURE CHANGES DURING NORMAL TERM PREGNANCY: THE AVON LONGITUDINAL STUDY OF PARENTS AND CHILDREN (ALSPAC)}

doi:10.1136/jech.2011.142976j.1

${ }^{1} \mathrm{C}$ Macdonald-Wallis, ${ }^{*}{ }^{1} \mathrm{~K}$ Tilling, ${ }^{1} \mathrm{~A}$ Fraser, ${ }^{2} \mathrm{~S}$ M Nelson, ${ }^{1} \mathrm{D}$ A Lawlor. ${ }^{1}$ University of Bristol, Bristol, UK; ${ }^{2}$ University of Glasgow, Glasgow, UK

Objective It is unclear whether established risk factors for preeclampsia are related to blood pressure (BP) changes across pregnancy in women who do not develop pre-eclampsia. We investigated these associations.

Methods We studied routine antenatal BP measurements for 11789 women (median 14 per woman) in ALSPAC who had a live term birth without pre-eclampsia or previous hypertension. Linear spline random effects models with knots at 18, 30 and 36 weeks gestation described changes in BP with gestational age.

Results On average systolic BP (SBP) and diastolic BP (DBP) decreased until 18 weeks and then rose, with increasing rate at 30 and then again at 36 weeks. BP was higher at 8 weeks for obese women compared with normal weight women, nulliparas compared with multiparas, and for never-smokers compared with women who smoked throughout pregnancy or in the first trimester only. Women who smoked throughout pregnancy had consistently lower BP while women who smoked only in the first trimester attained a similar BP trajectory to never-smokers in late pregnancy. BP rose more slowly between 18 and 30 weeks and more rapidly between 30 and 36 weeks in obese women than in normal weight women. Nulliparous women had faster rises in DBP from 30 weeks and SBP from 36 weeks than multiparous women and twin pregnancies were associated with faster rises in BP from 30 weeks.

Conclusion Established pre-eclampsia risk factors are associated with the pattern of BP changes in healthy pregnancy, suggesting that they affect BP change across the whole distribution.

\section{P2-167 MENTAL ILLNESS RELATED DISPARITIES IN DIABETES PREVALENCE, QUALITY OF CARE AND OUTCOMES: A POPULATION-BASED LONGITUDINAL STUDY IN WESTERN AUSTRALIA FROM 1990 TO 2006}

doi:10.1136/jech.2011.142976j.2

0 Mai, ${ }^{*}$ D Holman, F Sanfilippo, J Emery, D Preen. The University of Western Australia, Crawley, Western Australia, Australia

Introduction To compare the prevalence of diabetes, quality of care and outcomes between mental health clients (MHCs) and nonMHCs.

Methods Population-based longitudinal study of 139208 MHCs and 294180 matched non-MHCs in Western Australia (WA) from 1990 to 2006, using linked data of WA State mental health registry, electoral roll registrations, hospital admissions, emergency department attendances, deaths, and Commonwealth Medicare and pharmaceutical benefits claims. Diabetes was identified from hospital diagnoses, prescriptions and diabetes-specific primary care claims (17045 MHCs, 26626 non-MHCs). Prevalence of diabetes; likelihood of receiving recommended pathology tests for ongoing diabetes monitoring; risks of hospitalisation for diabetes complications, diabetes-related mortality and all-cause mortality.

Results Age-sex-standardised point-prevalence of diabetes in those aged 20 years was higher in MHCs than in non-MHCs $(9.3 \%$ vs $6.1 \%, p<0.001)$. The OR was 1.40 (95\% CI 1.36 to 1.43 ) after controlling for sociodemographics and case mix. Receipt of recom- mended pathology tests $\left(\mathrm{HbA}_{1 \mathrm{c}}\right.$, microalbuminuria, blood lipids) was suboptimal in both groups, but was even lower in MHCs (at 1 year, adjusted OR, 0.81 ; $95 \%$ CI 0.78 to 0.85 ; during the entire follow-up, adjusted rate ratio (RR), $0.86,95 \%$ CI 0.84 to 0.88 ; for all tests combined). MHCs also had increased risks of hospitalisation for diabetes complications (adjusted RR, 1.20, 95\% CI 1.17 to 1.24), diabetes-related mortality $(1.43,1.35$ to 1.52$)$ and all-cause mortality (1.47, 1.42 to 1.53$)$. The disparities were most marked for alcohol/ drug disorders, schizophrenia, affective disorders, other psychoses and personality disorders.

Conclusion MHCs require improved prevention and control of diabetes, especially at the primary care level.

\section{P2-168 MENTAL ILLNESS RELATED DISPARITIES IN POTENTIALLY PREVENTABLE HOSPITALISATIONS: A POPULATION-BASE COHORT STUDY FROM 1990 TO 2006}

doi:10.1136/jech.2011.142976j.3

Q Mai, ${ }^{*}$ D Holman, F Sanfilippo, J Emery. The University of Western Australia, Crawley, Australia

Introduction Emerging evidence indicates the association between mental illness and poor quality of physical healthcare. To test this, we compared mental health clients (MHCs) with non-MHCs for the quality of primary care, using potentially preventable hospitalisations (PPHs) as an indicator.

Methods Population-based retrospective cohort study of 139208 MHCs and 294180 matched non-MHCs in Western Australia from 1990 to 2006, using linked data of electoral roll registrations, mental health registry, hospital admissions and deaths. We used the electoral roll as the sampling frame for both cohorts to enhance internal validity and the mental health registry to separate MHCs from nonMHCs. Rates of first PPHs (overall and by PPH categories and conditions) were compared between MHCs, type of mental disorders and non-MHCs. Both unadjusted and adjusted analyses controlled for sociodemographic factors and case mix were performed using Cox regression models.

Results PPHs accounted for $>43 \%$ of all hospital admissions in both cohorts, predominantly chronic PPHs (88\% in MHCs and $90 \%$ in non-MHCs). MHCs with any mental disorders were more likely to be hospitalised for any PPH conditions than non-MHCs (overall adjusted rate ratio (ARR), $1.14 ; 95 \%$ CI 1.13 to 1.16). ARRs were highest for chronic PPHs $(1.18,1.16$ to 1.21$)$, especially congestive heart failure $(1.27,1.21$ to 1.32$)$, chronic obstructive pulmonary disease $(1.24,1.18$ to 1.31$)$ and diabetes complications $(1.19,1.15$ to 1.23); and highest for other psychoses (1.51, 1.46 to 1.56), alcohol/ drug disorders (1.34, 1.29 to 1.39$)$, schizophrenia $(1.15,1.09$ to 1.21$)$ and depressive disorders (1.14, 1.08 to 1.19).

Conclusion People with mental illness deserve special attention in improving the quality of physical healthcare at primary care level.

\section{P2-169 DO MENTAL HEALTH CLIENTS LACK ACCESS TO GENERAL PRACTITIONER SERVICES?}

doi:10.1136/jech.2011.142976j.4

Q Mai, ${ }^{*}$ D Holman, F Sanfilippo, J Emery. The University of Western Australia, Crawley, Australia

Introduction Emerging evidence indicates an association between mental illness and low-intensity of physical healthcare. To test this, we compared rates of visits to a general practitioner (GP) between mental health clients (MHCs) and non-MHCs.

Methods Population-based retrospective cohort study of 204727 MHCs and 294076 matched non-MHCs in Western Australia from 1 